Pacific Journal of Mathematics

AVERAGING PROPERTIES OF PLURIHARMONIC BOUNDARY 


\title{
AVERAGING PROPERTIES OF PLURIHARMONIC BOUNDARY VALUES
}

\author{
WADE C. RAMEY
}

\begin{abstract}
Suppose $D \subset C^{n}$ is a smoothly bounded domain and $u$ is bounded and pluriharmonic in $D$. Let $u^{*}$ denote the boundary function of $u$, and let $\zeta_{0} \in \partial D$. It is shown that if $u^{*}$ has good averaging behavior on one curve in $\partial D$ through $\zeta_{0}$, then $u^{*}$ has good averaging behavior on all curves in $\partial D$ through $\zeta_{0}$, provided the curves in question satisfy a certain directional condition. These results fail if the directional condition on the curve is violated.
\end{abstract}

I. Introduction. Let $D$ be a domain in $\mathbf{C}^{n}$ with $C^{1}$-boundary, and for $\zeta \in \partial D$, let $T_{\partial D}^{C}(\zeta)$ denote the complex tangent space of $\partial D$ at $\zeta$. If $f$ is a complex valued function defined in $D$, we denote by $f^{*}(\zeta)$ the nontangential limit of $f$ at $\zeta$, provided this limit exists.

Fix a point $\zeta_{0} \in \partial D$. We will be interested in $C^{3}$-curves $\gamma:(-1,1) \rightarrow$ $\partial D$ such that

$$
\gamma(0)=\zeta_{0}, \quad \gamma^{\prime}(0) \notin T_{\partial D}^{C}\left(\zeta_{0}\right) .
$$

Note that since $T_{\partial D}^{C}\left(\zeta_{0}\right)$ is of (real) codimension 1 within the full tangent space of $\partial D$ at $\zeta_{0}$, the "typical" smooth curve in $\partial D$ through $\zeta_{0}$ will satisfy the last condition in (1).

The results of this paper are concerned with averaging properties of pluriharmonic boundary values along such curves. The main thrust of these results is that if good averaging behavior occurs on one curve satisfying (1), then the same must be true of every curve satisfying (1). We first take up the case of $H^{\infty}$-boundary values.

THEOREM 1. If $f \in H^{\infty}(D)$, and if

$$
\lim _{h \rightarrow 0^{+}} \frac{1}{h} \int_{0}^{h} f^{*}(\gamma(x)) d x=L
$$

for one curve $\gamma$ satisfying (1), then (2) is true for every curve $\gamma$ satisfying (1).

Note that Theorem 1 also tells us that if (2) is true, then

$$
\lim _{h \rightarrow 0^{+}} \frac{1}{h} \int_{-h}^{0} f^{*}(\gamma(x)) d x=L
$$


as well, since the curve $\tilde{\gamma}$, defined by $\tilde{\gamma}(x)=\gamma(-x)$, satisfies (1) if and only if $\gamma$ satisfies (1).

An easy corollary of Theorem 1 generalizes the familiar one variable fact that the boundary values of an $H^{\infty}$-function cannot have a jump discontinuity.

COROLlaRY. If $\gamma_{1}$ and $\gamma_{2}$ are two curves satisfying (1), if $f \in H^{\infty}(D)$, and if

$$
\lim _{h \rightarrow 0^{+}}\left\|f^{*} \circ \gamma_{j}-L_{j}\right\|_{L^{\infty}(0, h)}=0, \quad j=1,2,
$$

then $L_{1}=L_{2}$.

1.2. The first thing one might wonder about in Theorem 1 is the existence of the boundary values $f^{*}(\gamma(x))$. It turns out that with $\gamma$ as in (1), $f^{*}(\gamma(x))$ is guaranteed to exist for a.e. $x$ in in a sufficiently small neighborhood of 0 , by the following result of Nagel and Rudin [7]:

Theorem A. Suppose $\varepsilon>0$, and that $\gamma:[-\varepsilon, \varepsilon] \rightarrow \partial D$ is a $C^{3}$-curve satisfying $\left\langle\gamma^{\prime}(x), \nu(\gamma(x))\right\rangle \neq 0$ for all $x \in[-\varepsilon, \varepsilon]$, where $\nu(\zeta)$ denotes the outward unit normal to $\partial D$ at $\zeta$, and $\langle$,$\rangle is the usual complex inner$ product. If $f \in H^{\infty}(D)$, then $f^{*}(\gamma(x))$ exists for a.e. $x \in[-\varepsilon, \varepsilon]$.

To see how Theorem A applies to our situation with $\gamma$ as in (1), define $\alpha(x)=\left\langle\gamma^{\prime}(x), \nu(\gamma(x))\right\rangle$. Then $\alpha(0) \neq 0$, for this is precisely the last condition in (1). The continuity of $\alpha$ then shows $\alpha(x) \neq 0$ in $[-\varepsilon, \varepsilon]$ for some $\varepsilon>0$, so that $f^{*}(\gamma(x))$ exists a.e. in $[-\varepsilon, \varepsilon]$ by Theorem A.

REMARK. Theorem A is true for curves with less than $C^{3}$-smoothness; see [8] for the strongest result. We are assuming $\gamma$ is $C^{3}$ to keep the exposition as simple as possible. (Later, in Part IV, it will be convenient to assume $\gamma$ is $C^{6}$.)

\section{Proof of Theorem 1 and related results.}

2.1. One variable preliminaries: For $\varepsilon>0$, set $Q_{\varepsilon}=(-\varepsilon, \varepsilon) \times(0, \varepsilon)$. Theorem 1 hinges on the following one variable result:

THEOREM B. If $f \in H^{\infty}\left(Q_{\varepsilon}\right)$, then the following statements are equivalent:

(i) $\lim _{h \rightarrow 0^{+}} h^{-1} \int_{0}^{h} f^{*}(x) d x=L$ 
(ii) $\lim _{h \rightarrow 0^{+}} h^{-1} \int_{-h}^{0} f^{*}(x) d x=L$

(iii) $\lim _{y \rightarrow 0^{+}} f($ iy $)=L$.

Theorem B for the upper half plane follows from Corollaries 3.5, 3.6, and 3.7 in [14]; very similar results for the unit disc appear in [1]. Of course, conformal maps transfer Theorem B from one region to another, and in particular to our region $Q_{\varepsilon}$.

Lemma 2.1. Let $\phi \in C^{1} \cap L^{\infty}\left(Q_{\varepsilon}\right)$. For $z \in \mathrm{C}$ set

$$
\psi(z)=\frac{1}{2 \pi i} \int_{Q_{\varepsilon}} \frac{\phi(\zeta)}{\zeta-z} d \zeta \wedge d \bar{\zeta}
$$

Then $\psi \in C^{1}\left(Q_{\varepsilon}\right), \partial \psi / \partial \bar{z}=\phi$ in $Q_{\varepsilon}$, and $\psi$ is continuous on all of $\mathbf{C}$.

Proof. We are using the notation $\partial / \partial \bar{z}=1 / 2(\partial / \partial x+i \partial / \partial y)$. That $\psi \in C^{1}\left(Q_{\varepsilon}\right)$ and $\partial \psi / \partial \bar{z}=\phi$ in $Q_{\varepsilon}$ is well known; see [11], p. 339. The continuity of $\psi$ on all of $\mathbf{C}$ follows since $\psi$ is the convolution of $\phi \in L^{\infty}\left(Q_{\varepsilon}\right)$ with $1 / \zeta \in L_{\text {loc }}^{1}(\mathbf{C})$.

2.2. Proof of Theorem 1. The first part of this argument comes from [7], where we refer the reader for details. (The details are even easier here since we are dealing with $C^{3}$-curves.)

If $\gamma$ satisfies (1), then one of the vectors $\pm i \gamma^{\prime}(0)$ points transversally into $D$ from $\zeta_{0}$; suppose it is $i \gamma^{\prime}(0)$ which does so. For $x+i y \in(-1,1) \times$ $\mathbf{R}$, define $\Gamma(x+i y)=\gamma(x)+i y \gamma^{\prime}(x)$. Since $i \gamma^{\prime}(0)$ points transversally into $D$ and $\Gamma(x)=\gamma(x)$ for $x \in(-1,1)$, there exists an $\varepsilon>0$ such that $\Gamma\left(Q_{\varepsilon}\right) \subset D$, and such that $d(\Gamma(x+i y), \partial D)$ is on the order of $|y|$ for $x+i y \in Q_{\varepsilon}$. Here $d(w, \partial D)$ denotes the Euclidean distance from $w \in \mathbf{C}^{n}$ to $\partial D$.

For $f \in H^{\infty}(D)$, the chain rule gives

$$
\frac{\partial(f \circ \Gamma)}{\partial \bar{z}}(x+i y)=\sum_{k=1}^{n} \frac{\partial f}{\partial w_{k}}(\Gamma(x+i y)) \frac{\partial \Gamma_{k}}{\partial \bar{z}}(x+i y)
$$

$\left(x+i y \in Q_{\varepsilon}\right)$, where we have written $\Gamma=\left(\Gamma_{1}, \ldots, \Gamma_{n}\right)$. Just as in [7], Cauchy's estimates show there is a constant $C$ such that

$$
\left|\partial f / \partial w_{k}(\Gamma(x+i y))\right| \leq C /|y| \text { for } x+i y \in Q_{\varepsilon},
$$

and we easily compute $\partial \Gamma / \partial \bar{z}(x+i y)=1 / 2 i y \gamma^{\prime \prime}(x)$. Thus by (3), $\partial(f \circ \Gamma) / \partial \bar{z} \in C^{1} \cap L^{\infty}\left(Q_{\varepsilon}\right)$.

Note that if instead it is $-i \gamma^{\prime}(0)$ which points into $D$, everything said so far holds verbatim with $-Q_{\varepsilon}$ in place of $Q_{\varepsilon}$. 
Now suppose $\gamma_{1}, \gamma_{2}$ satisfy (1), and that (2) holds for $\gamma_{1}$ and $f \in H^{\infty}(D)$. We assume that both $i \gamma_{1}^{\prime}(0)$ and $i \gamma_{2}^{\prime}(0)$ point transversally into $D$, the argument for the other cases being exactly the same. Associate $\Gamma_{j}$ to $\gamma_{j}$ on $Q_{\varepsilon}, j=1,2$, as described above. Since $\partial\left(f \circ \Gamma_{j}\right) / \partial \bar{z} \in C^{1} \cap$ $L^{\infty}\left(Q_{\varepsilon}\right)$, we can define

$$
\psi_{j}(z)=\frac{1}{2 \pi i} \int_{Q_{\varepsilon_{j}}} \frac{\partial\left(f \circ \Gamma_{j}\right) / \partial \bar{\zeta}}{\zeta-z} d \zeta \wedge d \bar{\zeta} \quad(x \in \mathbf{C})
$$

as in Lemma 2.1. We then have $f \circ \Gamma_{j}-\psi_{j} \in H^{\infty}\left(Q_{\varepsilon_{j}}\right)$. By the continuity of $\psi_{1}$ on $\mathbf{C}$,

$$
\lim _{h \rightarrow 0^{+}} \frac{1}{h} \int_{0}^{h}\left[f^{*}\left(\gamma_{1}(x)\right)-\psi_{1}(x)\right] d x=L-\psi_{1}(0) .
$$

By Theorem B this implies

$$
\lim _{y \rightarrow 0^{+}}\left(f \circ \Gamma_{1}-\psi_{1}\right)(i y)=L-\psi_{1}(0),
$$

which gives

$$
\lim _{y \rightarrow 0^{+}} f \circ \Gamma_{1}(i y)=L .
$$

But the curves $y \rightarrow \Gamma_{j}(i y)$ approach $\zeta_{0}$ nontangentially as $y \rightarrow 0^{+}$. From Črka's Lindelhöf theorem ([11], p. 168), we conclude

$$
\lim _{y \rightarrow 0^{+}} f \circ \Gamma_{2}(i y)=L \text {. }
$$

The above argument in reverse can now be applied to $f \circ \Gamma_{2}-\psi_{2}$ to obtain

$$
\lim _{h \rightarrow 0^{+}} \frac{1}{h} \int_{0}^{h} f^{*}\left(\gamma_{2}(x)\right) d x=L .
$$

REMARK. With some extra work, one can push the proof of Theorem 1 through to the case where $\gamma$ is $C^{1}$ and $\gamma^{\prime} \in \operatorname{Lip} \alpha$ for some $\alpha>1 / 2$, but it seems substantially more difficult to treat the case where $\gamma$ is merely $C^{1}$.

2.3. Weakly admissible limits. The notion of a weakly admissible limit was introduced in [3]; see also [9]. This coincides with the definition of hypoadmissible limit given in [2] and [5] and with the definition of restricted K-limit in [11]. Čirka's Lindelöf theorem ([3], and [11], p. 171), which we needed in the proof of Theorem 1, asserts that if $f \in H^{\infty}(D)$ and $f$ has a limit $L$ along a nontangential curve in $D$ terminating at $\zeta_{0} \in \partial D$, then $f$ has a weakly admissible limit $L$ at $\zeta_{0}$. 
TheOREM 2. If $f \in H^{\infty}(D)$, and $\partial D$ is $C^{3}$, then the following statements are equivalent:

(i) $\lim _{h \rightarrow 0^{+}} h^{-1} \int_{0}^{h} f^{*}(\gamma(x)) d x=L$ for one curve $\gamma$ satisfying (1).

(ii) $f$ has a weakly admissible limit $L$ at $\zeta_{0}$.

(iii) $\lim _{h \rightarrow 0^{+}} h^{-1} \int_{0}^{h} f^{*}(\gamma(x)) d x=L$ for every curve $\gamma$ satisfying (1).

Proof. Examining the proof of Theorem 1, we see that if $\gamma$ satisfies (1), and $\Gamma$ is associated with $\gamma$ as before, then

$$
\lim _{h \rightarrow 0^{+}} \frac{1}{h} \int_{0}^{h} f^{*}(\gamma(x)) d x=L \quad \text { iff } \quad \lim _{y \rightarrow 0^{+}} f(\Gamma(i y))=L .
$$

(More precisely, (4) is true if $i \gamma^{\prime}(0)$ points transversally into $D$ from $\zeta_{0}$; we assume without loss of generality that this is true of every $\gamma$ under consideration here.) As noted earlier, the curve $y \rightarrow \Gamma(i y)$ tends to $\zeta_{0}$ nontangentially as $y \rightarrow 0^{+}$. The implication (i) $\Rightarrow$ (ii) thus follows from Črka's Lindelöf theorem.

If (ii) holds, then

$$
\lim _{y \rightarrow 0^{+}} f(\Gamma(i y))=L
$$

for every $\gamma$ as above, since a weakly admissible limit implies a nontangential limit. Thus (ii) $\Rightarrow$ (iii) by (4).

Since $\partial D$ is $C^{3}$, there exist $C^{3}$-curves $\gamma$ in $\partial D$ satisfying (1), so that (iii) trivially implies (i).

2.4. Other averaging properties. Let $B_{n}$ denote the open unit ball in $\mathbf{C}^{n}$, and let $\sigma_{n}$ denote the usual rotation invariant Lebesgue measure on $\partial B_{n}$. If $\mu$ is a complex Borel measure on $\partial B_{n}$, we denote by $D \mu\left(\zeta_{0}\right)$ and $\mathscr{D} \mu\left(\zeta_{0}\right)$ the symmetric derivatives of $\mu$ at $\zeta_{0} \in \partial B_{n}$ defined in [10].

Specializing to the case $D=B_{n}$, we obtain

TheOREM 3. Let $f \in H^{\infty}\left(B_{n}\right)$, and put $d \mu=f^{*} d \sigma_{n}$. Then each of the statements $D \mu\left(\zeta_{0}\right)=L, \mathscr{D} \mu\left(\zeta_{0}\right)=L$ is equivalent to each statement of Theorem 2.

Proof. As shown in [10], the statements $D \mu\left(\zeta_{0}\right)=L$ and $\mathscr{D} \mu\left(\zeta_{0}\right)=L$ are each equivalent to the assertion

$$
\lim _{r \rightarrow 1} f\left(r \zeta_{0}\right)=L
$$

which is equivalent to (ii) of Theorem 2 for the case $D=B_{n}$ by Čirka's Lindelöf theorem. 


\section{Examples.}

3.1. Theorems 1 and 2 can fail in a variety of ways if the directional condition in (1) is violated. In the first place, the boundary values $f^{*}(\gamma(x))$ may not even exist. Suppose for example that $\partial D$ is $C^{2}$ and strictly pseudoconvex, and that $\gamma^{\prime}(x) \in T_{\partial D}^{C}(\gamma(x))$ for every $x \in(-1,1)$. Then as is well known, $\gamma([-1 / 2,1 / 2])$ is a peak set for $A(D)$, the algebra of functions continuous on $\bar{D}$ and holomorphic in $D$ (see, e.g., [11] p. 216). If $g \in A(D)$ peaks on $\gamma([-1 / 2,1 / 2])$, then $f=\exp [i \log (1-g)] \in$ $H^{\infty}(D)$, and $f^{*}(\gamma(x))$ fails to exist for every $x \in[-1 / 2,1 / 2]$. (Any logarithms, powers, or arguments appearing in this paper will always denote the usual principal branches.)

But even if $f^{*}(\gamma(x))$ exists for every $x \in(-1,1)$, Theorems 1 and 2 can fail rather badly if (1) is not satisfied. For convenience, we work in the unit ball $B$ in $\mathbf{C}^{2}$, with $\zeta_{0}=e_{1}=(1,0)$. Suppose $\gamma:(-1,1) \rightarrow \partial B$ is a $C^{2}$-curve such that

$$
\gamma(0)=e_{1}, \quad \gamma^{\prime}(0) \in T_{\partial B}^{C}\left(e_{1}\right), \quad \text { and } \quad \gamma^{\prime}(0) \neq 0 .
$$

(Note that (5) can be true while at the same time there exists an $\varepsilon>0$ such that $f^{*}(\gamma(x))$ exists a.e. in $(-\varepsilon, \varepsilon)$ for every $f \in H^{\infty}(D)$. This will happen if $\gamma^{\prime}(x) \notin T_{\partial D}^{C}(\gamma(x))$ in a deleted neighborhood of 0 , by Thm. A.) From (5) it follows that $\gamma(x)=\left(1+a x^{2}+o\left(x^{2}\right), b x+o(x)\right)$ as $x \rightarrow 0$, where $a, b \in \mathbf{C}$ and $b \neq 0$. The fact that $|\gamma(x)|=1$ for every $x \in(-1,1)$ implies $\operatorname{Re} a<0$. Define $f(z, w)=w(1-z)^{-1 / 2}$. Since $\left|w(1-z)^{-1 / 2}\right|^{2} \leq$ $|w|^{2}(1-|z|)^{-1} \leq 2$ for $(z, w) \in B, f \in H^{\infty}(B) \in C\left(\bar{B}-\left\{e_{1}\right\}\right)$. One easily computes

$$
\lim _{x \rightarrow 0^{+}} f^{*}(\gamma(x))=b(-a)^{-1 / 2}, \quad \lim _{x \rightarrow 0^{-}} f^{*}(\gamma(x))=-b(-a)^{-1 / 2} .
$$

Thus $f^{*}$ has a jump discontinuity along every curve satisfying (5); i.e., the corollary to Theorem 1 fails for all of these curves. Also note that this jump discontinuity depends on $a$ and $b$, i.e., the jump discontinuity varies from curve to curve. Finally, observe that $f$ has a weakly admissible limit 0 at $e_{1}$.

3.2. Here we give an example of an $f \in H^{\infty}(B)$ with weakly admissible limit at $e_{1}$, but such that

$$
\lim _{h \rightarrow 0^{+}} \frac{1}{h} \int_{0}^{h} f^{*}(\gamma(x)) d x
$$

fails to exist for every $\gamma$ satisfying (5). 
For $z \in U$, the open unit disc in $\mathbf{C}$, define $g(z)=\exp [i \log (1-z)]$. Clearly $g \in H^{\infty}(U) \cap C(\bar{U}-\{1\})$. For $(z, w) \in B$, define $f(z, w)=$ $w^{2}(1-z)^{-1} g(z)$. As in 3.1 one verifes $f \in H^{\infty}(B) \cap C\left(\bar{B}-\left\{e_{1}\right\}\right)$, and clearly $f$ has a weakly admissible limit 0 at $e_{1}$. We claim that for every $\gamma$ as in (5),

$$
\lim _{h \rightarrow 0^{+}} \frac{1}{h} \int_{0}^{h} f^{*}(\gamma(x)) d x \quad \text { fails to exist. }
$$

Writing out the components of $\gamma$ as in 3.1, and noting

$$
\lim _{x \rightarrow 0}(b x+o(x))^{2}\left(-a x^{2}+o\left(x^{2}\right)\right)^{-1}=b^{2}(-a)^{-1} \neq 0,
$$

it is clear that to prove (6) we need only look at

$$
\frac{1}{h} \int_{0}^{h} g\left(1+a x^{2}+o\left(x^{2}\right)\right) d x .
$$

Since $g \in H^{\infty}(U),\left|g^{\prime}(z)\right|(1-|z|)$ is bounded in $U$, hence

$$
\lim _{x \rightarrow 0}\left[g\left(1+a x^{2}+o\left(x^{2}\right)\right)-g\left(1+a x^{2}\right)\right]=0 .
$$

(As $x \rightarrow 0,1+a x^{2}$ approaches 1 nontangentially since $\operatorname{Re} a<0$.) Thus it is enough to consider

$$
\frac{1}{h} \int_{0}^{h} g\left(1+a x^{2}\right) d x .
$$

This latter integral equals

$$
\frac{1}{h} \int_{0}^{h} e^{i \log \left(-a x^{2}\right)} d x=\int_{0}^{1} e^{i \log \left(-a h^{2} x^{2}\right)} d x .
$$

Since $\arg \left(-a h^{2} x^{2}\right)$ is constant, (7) is equal to

$$
e^{-\arg (-a)} e^{i \log \left|a h^{2}\right|} \int_{0}^{1} e^{i \log x^{2}} d x .
$$

The integral in (8) is not zero since (7) clearly does not vanish for all $h$. Thus (8) has no limit as $h \rightarrow 0^{+}$, proving (6).

3.3. In the other direction, we give an example of an $f \in H^{\infty}(B)$ and a $\gamma$ satisfying (5), such that $f^{*}$ is constant a.e. on $\gamma(-1,1)$, but such that $f^{*}\left(e_{1}\right)$ fails to exist. To do this we use the following theorem of Saerens [13]: if $K$ is a peak set for $A(B), \mu$ is a finite positive Borel measure on $K$, and $b \in L^{\infty}(\mu)$, then there exists $g \in H^{\infty}(B)$ such that

$$
\|g\|_{H^{\infty}(B)} \leq\|b\|_{L^{\infty}(\mu)}
$$

and $g^{*}=b \mu$-a.e. on $K$. 
If $\gamma:(-1,1) \rightarrow \partial B$ is any $C^{1}$-curve such that $\gamma(0)=e_{1}$ and $0 \neq \gamma^{\prime}(x)$ $\in T_{\partial B}^{c}(\gamma(x))$ for every $x \in(-1,1)$ (the curve $\gamma(x)=(\cos x, \sin x)$ will do), then as was mentioned earlier, $\gamma([-1 / 2,1 / 2])$ is a peak set for $A(B)$. Define $\mu=s+\delta$, where $s$ denotes arclength measure on $\gamma([-1 / 2,1 / 2])$, and $\delta$ is the unit point mass at $e_{1}$. Set $b(\gamma(x))=0, x \in[-1 / 2,1 / 2]-$ $\{0\}$, and $b\left(e_{1}\right)=1$. By Saerens's theorem, there exists a

$$
g \in H^{\infty}(B), \quad\|g\|_{H^{\infty}(B)} \leq 1,
$$

such that $g^{*}=b \mu$-a.e. on $\gamma([-1 / 2,1 / 2])$. Thus $g^{*}(\gamma(x))=0$ for a.e. $x \in[-1 / 2,1 / 2]$ and $g^{*}\left(e_{1}\right)=1$. Setting $f=\exp [i \log (1-g)]$, we see $f^{*}(\gamma(x))=1$ a.e. in $[-1 / 2,1 / 2]$, while $\lim _{r \rightarrow 1} f\left(r e_{1}\right)$ does not exist.

\section{The pluriharmonic case.}

4.1. Theorems 1 and 2 fail for bounded pluriharmonic functions, and for a simple reason: Theorem B is false for bounded harmonic functions. (Example: $u(z)=\arg z$ ). There is, however, a substitute for Theorem B (Theorem $\mathrm{C}$ below), involving symmetric averages, which will enable us to prove the following theorem.

THeOREM 4. If $u$ is bounded and pluriharmonic in $D$, and $\partial D$ is $C^{6}$, then the following statements are equivalent:

(i) $\lim _{h \rightarrow 0^{+}}(1 / 2 h) \int_{-h}^{h} u^{*}(\gamma(x)) d x=L$ for one $C^{6}$-curve $\gamma$ satisfying (1).

(ii) $\lim _{y \rightarrow 0^{+}} u\left(\zeta_{0}-y \nu\left(\zeta_{0}\right)\right)=L$.

(iii) $\lim _{h \rightarrow 0^{+}}(1 / 2 h) \int_{-h}^{h} u^{*}(\gamma(x)) d x=L$ for every $C^{6}$-curve $\gamma$ satisfying (1).

REMARKs. 1 . The existence of the boundary values $u^{*}(\gamma(x))$ for a.e. $x$ in a small enough interval about 0 follows easily from Theorem A. Fix a curve $\gamma$ satisfying (1) and assume without loss of generality that $u$ is real. Since there exists a neighborhood $V$ of $\zeta_{0}$ (open in $\mathbf{C}^{n}$ ) such that $V \cap D$ is simply connected, there exists a pluriharmonic $v$ in $V \cap D$ such that $u+i v$ is holomorphic in $V \cap D$. The function $f=e^{u+i v}$ then belongs to $H^{\infty}(V \cap D)$, which implies $f^{*}(\gamma(x))$ exists a.e. in $[-\varepsilon, \varepsilon]$ for some $\varepsilon>0$ as in 1.2. Clearly $u^{*}(\gamma(x))$ exists wherever $f^{*}(\gamma(x))$ exists.

2. In proving Theorem 4 we will need to deal with the operator $\Delta=\partial^{2} / \partial x^{2}+\partial^{2} / \partial y^{2}$ rather than $\partial / \partial \bar{z}$. Thus twice as many derivatives will be involved here, accounting for the assumption $\gamma \in C^{6}$ rather than $\gamma \in C^{3}$. 
4.2. Theorem $\mathrm{C}$ and Lemma 4.2 below will play the same roles in proving Theorem 4, respectively, as Theorem B and Lemma $2.1 \mathrm{did}$ in proving Theorems 1 and 2.

THEOREM C. If $u$ is bounded and harmonic in $Q_{\varepsilon}$, then the following statements are equivalent:

(i) $\lim _{h \rightarrow 0^{+}}(1 / 2 h) \int_{-h}^{h} u^{*}(x) d x=L$

(ii) $\lim _{y \rightarrow 0^{+}} u($ iy $)=L$.

Theorem $\mathrm{C}$ for the upper half plane is due to Loomis [6]; again, a conformal map transfers this to $Q_{\varepsilon}$. (For generalizations of Theorem $\mathrm{C}$ to the upper half space of $\mathbf{R}^{n}$, see [12]. Versions of Theorem $C$ for the unit ball in $C^{n}$ were obtained in [10]. All of these results hold under the weaker hypothesis that $u$ is positive.)

Lemma 4.2. Suppose $\phi \in C^{2} \cap L^{\infty}\left(Q_{\varepsilon}\right)$. For $z \in \mathbf{C}$, define

$$
\psi(z)=\frac{1}{2 \pi} \int_{Q_{\varepsilon}} \phi(\zeta) \log |z-\zeta| d x d y \quad(\zeta=x+i y) .
$$

Then $\psi \in C^{2}\left(Q_{\varepsilon}\right), \Delta \psi=\phi$ in $Q_{\varepsilon}$, and $\psi$ is continuous on all of $\mathbf{C}$.

Proof. The first two assertions are well known; see [5], pp. 29-30. That $\psi$ is continuous on all of $\mathbf{C}$ follows as in Lemma 2.1: $\psi$ is the convolution of $\phi \in L^{\infty}\left(Q_{\varepsilon}\right)$ with $\log |\zeta| \in L_{\mathrm{loc}}^{1}(\mathbf{C})$.

4.3. It will be convenient in the rest of the paper to suppose $\zeta_{0}=0$ and $\nu\left(\zeta_{0}\right)=(-i, 0, \ldots, 0)$; recall that $\nu\left(\zeta_{0}\right)$ is the outward unit normal to $\partial D$ at $\zeta_{0}$. We let $\mathbf{C}_{+}^{n}$ denote the half space $\left\{z=\left(z_{1}, \ldots, z_{n}\right) \in \mathbf{C}^{n}\right.$ : $\left.\operatorname{Im} z_{1}>0\right\}$. Note that with these conventions, $\mathbf{C}_{+}^{n}$ can be thought of as the set of vectors pointing into $D$ transversally from $\zeta_{0}=0$.

Define $Y=\left\{\left(i y, z^{\prime}\right): y>0, z^{\prime} \in \mathbf{C}^{n-1}\right\}$. In Lemma 4.3, which is an analogue of Čirka's Lindelöf theorem, we consider $C^{1}$-curves $\eta$ such that

$$
\eta:[0,1] \rightarrow \bar{D}, \quad \eta(0)=0, \quad \eta^{\prime}(0) \in Y .
$$

LEMMA 4.3. If $u$ is bounded and pluriharmonic in $D$, and if

$$
\lim _{y \rightarrow 0^{+}} u(\eta(y))=L
$$

for one curve $\eta$ satisfying (9), then

$$
\lim _{y \rightarrow 0^{+}} u(\eta(y))=L
$$

for every curve $\eta$ satisfying (9). 
Proof. The proof is very much like the proof of Čirka's Lindelöf theorem, and rests on two well known properties of $\nabla u$. First, the boundedness of $u$ implies the boundedness of $|\nabla u(z)| d(z, \partial D)$ for $z \in D$. Second, there is a better estimate on the derivatives of $u$ in certain directions: if $\nabla_{T} u$ denotes the gradient of $u$ with respect to the variables $x_{2}, y_{2}, \ldots, x_{n}, y_{n}$ (i.e., the gradient of $u$ in the complex tangential directions), then $\left|\nabla_{T} u(z)\right| d(z, \partial D) \rightarrow 0$ as $z \rightarrow 0$ in $D$ nontangentially. (See, e.g., inequality (20) of [9].)

From the first property it follows that

$$
\lim _{y \rightarrow 0^{+}} u(\eta(y))=L
$$

if and only if

$$
\lim _{y \rightarrow 0^{+}} u\left(y \eta^{\prime}(0)\right)=L,
$$

for any curve $\eta$ as in (9). Thus we need only consider limits along rays contained in $Y$.

Suppose $v_{1}, v_{2} \in Y$, and that $\lim _{y \rightarrow 0^{+}} u\left(y v_{1}\right)=L$; we wish to show $\lim _{y \rightarrow 0^{+}} u\left(y v_{2}\right)=L$. The definition of $Y$ shows there exist $y_{0}>0$ and $z_{0} \in\{0\} \times \mathbf{C}^{n-1}$ such that $v_{2}=y_{0} v_{1}+z_{0}$, and we have the standard estimate

$$
\left|u\left(y v_{2}\right)-u\left(y y_{0} v_{1}\right)\right| \leq \sup _{\substack{z \in\{0\} \times C^{n-1} \\|z| \leq y\left|z_{0}\right|}}\left|\nabla_{T} u\left(y y_{0} v_{1}+z\right)\right|\left|y z_{0}\right| .
$$

For small $y>0$, the points $y y_{0} v_{1}+z$ satisfying $|z| \leq y\left|z_{0}\right|$ lie within a nontangential approach region, and $d\left(y y_{0} v_{1}+z, \partial D\right)$ is on the order of $y$. Thus the right hand side of $(10) \rightarrow 0$ as $y \rightarrow 0^{+}$by the second property mentioned above, so that $\lim _{y \rightarrow 0^{+}} u\left(y v_{2}\right)=L$ as desired.

4.4. Proof of Theorem 4. If $\gamma$ is any $C^{6}$-curve satisfying (1), we may assume that $i \gamma^{\prime}(0)$ points transversally into $D$ from 0 , since the symmetric averages in (i) and (iii) are unaffected if $\gamma(x)$ is replaced by $\gamma(-x)$. For any such $\gamma$, define

$\Gamma(x+i y)=\gamma(x)+i y \gamma^{\prime}(x)-\left(y^{2} / 2\right) \gamma^{\prime \prime}(x) \quad(x+i y \in(-1,1) \times \mathbf{R})$.

Then for some $\varepsilon>0, \Gamma$ has the same properties on $Q_{\varepsilon}$ as did the earlier $\Gamma$ in the proof of Theorem 1, except this time $\partial \Gamma / \partial \bar{z}(x+i y)=$ $-1 / 4 \gamma^{\prime \prime \prime}(x) y^{2}$.

If $u$ is bounded and pluriharmonic in $D$, we claim

$$
\Delta(u \circ \Gamma) \in C^{2} \cap L^{\infty}\left(Q_{\varepsilon}\right) .
$$


To calculate $\Delta(u \circ \Gamma)$ it is best to write $\Delta=4 \partial^{2} / \partial z \partial \bar{z}$, and use the $\partial / \partial \bar{z}$ version of the chain rule. This slightly tedious computation was carried out in the proof of Theorem 1 in [9], and we spare the reader the details here. Once $\Delta(u \circ \Gamma)$ has been computed, (11) follows from Cauchy-type estimates on the derivatives of $u$, the vanishing of $\partial^{2} u / \partial z_{i} \partial \bar{z}_{j}$ for all $i$ and $j$, and the second order vanishing of $\partial \Gamma / \partial \bar{z}$ on $(-\varepsilon, \varepsilon)$. (Note that second order derivatives of $\Gamma$ appear in the expression for $\Delta(u \circ \Gamma)$. Since the definition of $\Gamma$ already involves two derivatives of $\gamma$, we see that to assert $\Delta(u \circ \Gamma) \in C^{2}\left(Q_{\varepsilon}\right)$ requires that $\gamma$ be $C^{6}$.)

Suppose now that (i) holds for $u$ and $\gamma$. With $\Gamma$ defined as above, we see by (11) that $\psi$ can be defined as in Lemma 4.2, taking $\phi=\Delta(u \circ \Gamma)$. The function $u \circ \Gamma-\psi$ is then bounded and harmonic in $Q_{\varepsilon}$, and by Theorem $\mathbf{C}$ we conclude, since $\psi$ is continuous in $\mathbf{C}$, that

$$
\lim _{y \rightarrow 0^{+}} u(\Gamma(i y))=L \quad \text { iff } \quad \lim _{h \rightarrow 0^{+}} \frac{1}{2 h} \int_{-h}^{h} u^{*}(\gamma(x)) d x=L .
$$

Setting $\eta(y)=\Gamma(i y)$, it is easily verified that $\eta$ satisfies (9). Since the curve $y \rightarrow \zeta_{0}-y \nu\left(\zeta_{0}\right)=(i y, 0, \ldots, 0)$ obviously satisfies (9), Lemma 4.3 shows that (ii) must hold.

A similar argument based on (12) and Lemma 4.3 shows that (ii) $\Rightarrow$ (iii), and the implication (iii) $\Rightarrow$ (i) is obvious since $\partial D$ is $C^{6}$.

4.5. Specializing to the ball, we have the following analogue of Theorem 3.

THEOREM 5. Suppose $u$ is bounded and pluriharmonic in $B_{n}$, and put $d \mu=u^{*} d \sigma_{n}$. Then each of the statements $D \mu\left(\zeta_{0}\right)=L, \mathscr{D} \mu\left(\zeta_{0}\right)=L$ is equivalent to each statement of Theorem 4.

Proof. Again, each of the statements $D \mu\left(\zeta_{0}\right)=L, \mathscr{D} \mu\left(\zeta_{0}\right)=L$ is shown in [10] to be equivalent to the statement $\lim _{r \rightarrow 1^{-}} u\left(r \zeta_{0}\right)=L$.

4.6. Differentiability along $\gamma$ and weakly admissible limits. Unlike the case for an $H^{\infty}$-function, the equivalent statements of Theorem 4 do not imply a weakly admissible limit for a bounded pluriharmonic function. The simple example $u\left(z_{1}, z_{2}\right)=\arg \left(1-z_{1}\right)$ in $B_{2}$ (take $\left.\zeta_{0}=e_{1}\right)$ shows this. What is needed is a stronger differentiability assumption on $u^{*}$ along $\gamma$.

First we give some definitions. If $\gamma$ is a curve satisfying (1), if $\varepsilon$ is chosen as in 1.2, and if $u$ is bounded and pluriharmonic in $D$, define

$$
U_{\gamma}(t)=\int_{-\varepsilon}^{t} u^{*}(\gamma(x)) d x \quad(t \in(-\varepsilon, \varepsilon)) .
$$


For $z=\left(z_{1}, \ldots, z_{n}\right) \in \mathbf{C}_{+}^{n}$, define $a(z)=\arg z_{1}$; clearly $0<a(z)<\pi$ for all $z \in \mathbf{C}_{+}^{n}$.

THEOREM 6. If $u$ is bounded and pluriharmonic in $D$, and $\partial D$ is $C^{6}$, then the following statements are equivalent:

(i) there exists a $C^{6}$-curve $\gamma$ satisfying (1) such that $U_{\gamma}^{\prime}(0)=L$.

(ii) there exist $v_{1}, \quad v_{2} \in \mathbf{C}_{+}^{n}$, with $a\left(v_{1}\right) \neq a\left(v_{2}\right)$, such that $\lim _{r \rightarrow 0^{+}} u\left(r v_{1}\right)=L=\lim _{r \rightarrow 0^{+}} u\left(r v_{2}\right)$.

(iii) $u$ has a weakly admissible limit $L$ at 0 .

(iv) $U_{\gamma}^{\prime}(0)=L$ for every $C^{6}$-curve $\gamma$ satisfying (1).

As was the case earlier, the proof of Theorem 6 strongly depends on a one variable result:

THEOREM D. If $u$ is bounded and harmonic in $Q_{\varepsilon}$, put

$$
U(t)=\int_{-\varepsilon}^{t} u^{*}(x) d x \quad(t \in(-\varepsilon, \varepsilon)) .
$$

Then the following statements are equivalent:

(i) $U^{\prime}(0)=L$.

(ii) There exist $\theta_{1}, \theta_{2}, 0<\theta_{1}<\theta_{2}<\pi$, such that $\lim _{r \rightarrow 0^{+}} u\left(r e^{i \theta_{j}}\right)=$ $L, j=1,2$.

(iii) $u$ has a nontangential limit $L$ at 0 .

Theorem D, proved for the upper half plane, is due to Loomis [6]. See also Gehring [4].

Proof of Theorem 6. As usual, we assume without loss of generality that if $\gamma$ satisfies (1), then $i \gamma^{\prime}(0)$ points transversally into $D$ from 0 . Since the reader by now is familiar with our strategy of proof, we will be brief about certain details.

Suppose (i) holds for $u$ and $\gamma$. Associating $\Gamma$ with $\gamma$ as in the proof of Theorem 4, and using our usual argument together with Theorem $\mathrm{D}$, we see that $u \circ \Gamma$ has a nontangential limit $L$ at 0 . So certainly there exist $\theta_{1}$, $\theta_{2}, 0<\theta_{1}<\theta_{2}<\pi$, such that

$$
\lim _{r \rightarrow 0^{+}} u\left(\Gamma\left(r e^{i \theta_{j}}\right)\right)=L, \quad j=1,2 .
$$

The fact that $\partial \Gamma / \partial \bar{z}(0)=0$ implies the curves $r \rightarrow \Gamma\left(r e^{\imath \theta_{j}}\right)$ are tangent to the rays $r e^{i \theta} \gamma^{\prime}(0), j=1,2$, respectively. Thus

$$
\lim _{r \rightarrow 0^{+}} u\left(r e^{i \theta,} \gamma^{\prime}(0)\right)=L, \quad j=1,2 .
$$


Since

$$
a\left(e^{i \theta_{j}} \gamma^{\prime}(0)\right)=\theta_{j}
$$

we see that (ii) holds.

In proving (ii) $\Rightarrow$ (iii) we need some preliminaries. For $0<\theta<\pi$, set $Y_{\theta}=\left\{\left(r e^{i \theta}, z\right): r>0, z \in \mathbf{C}^{n-1}\right\}$. (The $Y$ defined in $\$ 4.3$ is $Y_{\pi / 2}$ in the new notation.). If $\eta_{1}$ and $\eta_{2}$ are $C^{1}$-curves satisfying $\eta_{j}:[0,1] \rightarrow \bar{D}$, $\eta_{j}(0)=0, \eta_{j}^{\prime}(0) \in Y_{\theta}$, then

$$
\lim _{r \rightarrow 0^{+}} u\left(\eta_{1}(r)\right)=L \text { if and only if } \lim _{r \rightarrow 0^{+}} u\left(\eta_{2}(r)\right)=L \text {. }
$$

The proof of this is exactly the same as the proof of Lemma 4.3.

Now suppose (ii) holds, and put $\theta_{j}=a\left(v_{j}\right), j=1,2$. Let $N$ be the complex line $\{(\lambda, 0, \ldots, 0): \lambda \in \mathbf{C}\}$, and set $D_{N}=D \cap N$. The vectors $\left(e^{i \theta_{1}}, 0\right), v_{1}$ are both in $Y_{\theta_{1}}$, and similarly $\left(e^{i \theta_{2}}, 0\right), v_{2} \in Y_{\theta_{2}}$. We conclude

$$
\lim _{r \rightarrow 0^{+}} u\left(r e^{i \theta_{j}}, 0\right)=L, \quad j=1,2 \text {. }
$$

Since $D_{N}$ can be thought of as a domain in $\mathbf{C}^{1}$, and since Theorem D is true for any domain with smooth boundary, we conclude that $u$ has a nontangential limit within $D_{N}$ at 0 . Estimates on $\nabla_{T} u$ like the one mentioned in proving Lemma 4.3 now show that $u$ has a weakly admissible limit $L$ at 0 . (We omit the proof of this last statement; the argument is essentially the same as in the proof of Lemma 4.3. Again, see [3] or [9] for the definition of a weakly admissible limit.)

If (iii) holds, let $\gamma$ be a $C^{6}$-curve satisfying (1), and associate $\Gamma$ with $\gamma$ as before. If $K$ is a nontangential approach region in $Q_{\varepsilon}$ of small enough height, $\Gamma(K)$ will be contained in a nontangential approach region in $D$ with vertex 0 . Since a weakly admissible limit implies a nontangential limit, (iii) implies $u \circ \Gamma$ has a nontangential limit $L$ at 0 within $Q_{\varepsilon}$. Our usual argument, together with Theorem $\mathrm{D}$, then gives (iv).

Finally, since $\partial D$ is $C^{6}$, (iv) implies (i).

\section{REFERENCES}

[1] T. K. Boehme, M. Rosenfeld and Max L. Weiss, Relations between bounded analytic functions and their boundary functions, J. London Math. Soc., (2) 1 (1969), 609-618.

[2] J. A. Cima and S. G. Krantz, The Lindelöf principle and normal functions of several complex variables, Duke Math. J., 50 (1983), 303-328.

[3] E. M. Čirka, The theorems of Lindelöf and Fatou in $C^{n}$, Mat. Sb., 92 (1973), 622-644; Math. USSR-Sb., 21 (1973), 619-639.

[4] F. W. Gehring, Harmonic functions and tauberian theorems, Proc. London Math. Soc., (3) 10 (1960), 88-106. 
[5] S. G. Krantz, Function Theory of Several Complex Variables, John Wiley \& Sons Inc., New York, 1982.

[6] L. H. Loomis, The converse of the Fatou theorem for positive harmonic functions, Trans. Amer. Math. Soc., 53 (1943), 239-250.

[7] A. Nagel and W. Rudin, Local boundary behavior of bounded holomorphic functions, Canad. J. Math., 30 (1978). 583-592.

[8] A. Nagel and S. Wainger, Limits of bounded holomorphic functions along curves, Recent Developments in Several Complex Variables, Ann. Math. Studies 100, Princeton University Press, Princeton, NJ (1981), 327-344.

[9] W. Ramey, Local boundary behavior of pluriharmonic functions along curves, to appear in Amer. J. Math.

[10] W. Ramey and D. Ullich, The pointwise Fatou theorem and its converse for positive pluriharmonic functions, Duke Math. J., 49 (1982), 655-675.

[11] W. Rudin, Function Theory in the Unit Ball of $C^{n}$, Springer-Verlag, New York, 1980.

[12] Tauberian theorems for positive harmonic functions, Nederl. Akad. Wetensch. Proc., Ser. A. 81 (1978), 376-384.

[13] R. Saerens, Interpolation manifolds, Ann. Scuola Norm. Sup. Pisa. A. Sci., (4) 11 (1984), 177-211.

[14] H. S. Shapiro, Boundary values of bounded holomorphic functions of several variables, Bull. Amer. Math. Soc., 77 (1971), 111-116.

Received February 8, 1985 and in revised form September 25, 1985. This research was partially supported by NSF grant DMS-8405382.

Michigan State University

EAST LANSING, MI 48824 


\title{
PACIFIC JOURNAL OF MATHEMATICS EDITORS
}

\author{
V. S. VARADARAJAN \\ (Managing Editor) \\ University of California \\ Los Angeles, CA 90024 \\ HERBERT ClEMENS \\ University of Utah \\ Salt Lake City, UT 84112 \\ R. FINN \\ Stanford University \\ Stanford, CA 94305
}

\author{
HERMANN FLASCHKA \\ University of Arizona \\ Tucson, AZ 85721 \\ RAMESH A. GANGOLLI \\ University of Washington \\ Seattle, WA 98195 \\ VAUGHAN F. R. JONES \\ University of California \\ Berkeley, CA 94720 \\ ROBION KIRBY \\ University of California \\ Berkeley, CA 94720
}

C. C. MOORE

University of California

Berkeley, CA 94720

H. SAMELSON

Stanford University

Stanford, CA 94305

HAROLD STARK

University of California, San Diego

La Jolla, CA 92093

\section{ASSOCIATE EDITORS}

\author{
R. AREnS \\ E. F. BECKENBACH \\ B. H. NEUMANN \\ F. WOLF \\ K. YOSHIDA \\ (1906-1982)

\section{SUPPORTING INSTITUTIONS}

\begin{abstract}
UNIVERSITY OF ARIZONA
UNIVERSITY OF BRITISH COLUMBIA

UNIVERSITY OF CALIFORNIA

MONTANA STATE UNIVERSITY

UNIVERSITY OF NEVADA, RENO

NEW MEXICO STATE UNIVERSITY

OREGON STATE UNIVERSITY
\end{abstract} \\ CALIFORNIA INSTITUTE OF TECHNOLOGY \\ UNIVERSITY OF OREGON \\ UNIVERSITY OF SOUTHERN CALIFORNIA \\ STANFORD UNIVERSITY \\ UNIVERSITY OF HAWAII \\ UNIVERSITY OF TOKYO \\ UNIVERSITY OF UTAH \\ WASHINGTON STATE UNIVERSITY \\ UNIVERSITY OF WASHINGTON
}

The Supporting Institutions listed above contribute to the cost of publication of this Journal, but they are not owners or publishers and have no responsibility for its content or policies.

Mathematical papers intended for publication in the Pacific Journal of Mathematics should be in typed form or offset-reproduced (not dittoed), double spaced with large margins. Please do not use built up fractions in the text of the manuscript. However, you may use them in the displayed equations. Underline Greek letters in red, German in green, and script in blue. The first paragraph must be capable of being used separately as a synopsis of the entire paper. In particular it should contain no bibliographic references. Please propose a heading for the odd numbered pages of less than 35 characters. Manuscripts, in triplicate, may be sent to any one of the editors. Please classify according to the scheme of Math. Reviews, Index to Vol. 39. Supply name and address of author to whom proofs should be sent. All other communications should be addressed to the managing editor, or Elaine Barth, University of California, Los Angeles, California 90024.

There are page-charges associated with articles appearing in the Pacific Journal of Mathematics. These charges are expected to be paid by the author's University, Government Agency or Company. If the author or authors do not have access to such Institutional support these charges are waived. Single authors will receive 50 free reprints; joint authors will receive a total of 100 free reprints. Additional copies may be obtained at cost in multiples of 50 .

The Pacific Journal of Mathematics is issued monthly as of January 1966. Regular subscription rate: $\$ 190.00$ a year (5 Vols., 10 issues). Special rate: $\$ 95.00$ a year to individual members of supporting institutions.

Subscriptions, orders for numbers issued in the last three calendar years, and changes of address should be sent to Pacific Journal of Mathematics, P.O. Box 969, Carmel Valley, CA 93924, U.S.A. Old back numbers obtainable from Kraus Periodicals Co., Route 100, Millwood, NY 10546.

The Pacific Journal of Mathematics at P.O. Box 969, Carmel Valley, CA 93924 (ISSN 0030-8730) publishes 5 volumes per year. Application to mail at Second-class postage rates is pending at Carmel Valley, California, and additional mailing offices. Postmaster: send address changes to Pacific Journal of Mathematics, P.O. Box 969, Carmel Valley, CA 93924.

PUBLISHED BY PACIFIC JOURNAL OF MATHEMATICS, A NON-PROFIT CORPORATION

Copyright (C) 1986 by Pacific Journal of Mathematics 


\section{Pacific Journal of Mathematics}

\section{Vol. 123, No. $2 \quad$ April, 1986}

David Jay Anick, A loop space whose homology has torsion of all orders . . 257 Steven P. Diaz, Space curves that intersect often ................. 263

Thierry Fack and Hideki Kosaki, Generalized $s$-numbers of $\tau$-measurable

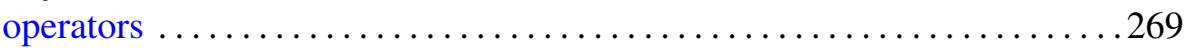

Karl Heinrich Hofmann and Karl Strambach, Lie's fundamental

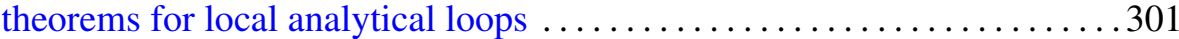

James Secord Howland, On the Kato-Rosenblum theorem ............. 329

Frieder Knüppel and Edzard Salow, Plane elliptic geometry over rings . . . . 337

Alan Noell, Peak points in boundaries not of finite type ................ 385

William J. Ralph, An extension of singular homology to Banach

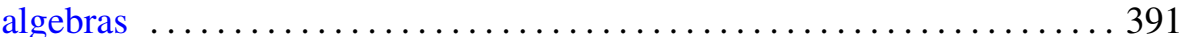

Wade C. Ramey, Averaging properties of pluriharmonic boundary values . . 407

Thomas Joseph Ransford, On the range of an analytic multivalued

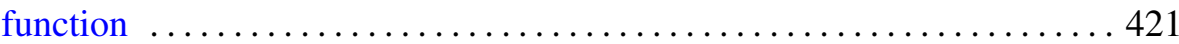

Christopher Donald Sogge, On restriction theorems of maximal-type . . . . 441

Edwin Spanier, Cohomology with supports $\ldots \ldots \ldots \ldots \ldots \ldots \ldots \ldots \ldots 47$

Emil J. Straube, Orthogonal projections onto subspaces of the harmonic

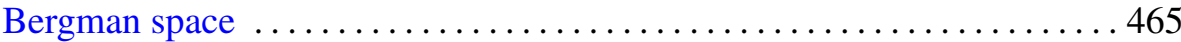

Thomas Vogel, Asymptotic behavior of two semilinear elliptic free boundary problems $\ldots \ldots \ldots \ldots \ldots \ldots \ldots \ldots \ldots$ 